\title{
Graphene: A Multifaceted Nanomaterial for Cutting Edge Biomedical Application
}

\author{
Reshma S Cherian and Mohanan PV*
}

Toxicology Division, Sree Chitra Tirunal Institute for Medical Sciences and Technology, India

*Corresponding author: Mohanan PV, Toxicology Division, Biomedical Technology Wing Sree Chitra Tirunal Institute for Medical Sciences and Technology, Poojapura, Trivandrum 695 012, Kerala, India, E-mail: mohanpv10@gmail.com

\begin{abstract}
Graphene, a discrete class of nanomaterial, has come into the limelight after its discovery in 2004. Its unique physico-chemical characteristics like electrical, optical, thermal properties and high mechanical strength has piqued the curiosity of the scientific community worldwide. These properties have been utilized for innovative and novel applications in the field of nanoelectronics and biomedicine. The review focuses on the opportunities and prospects of graphene in biomedical field mainly drug and gene delivery, photo thermal therapy, tissue engineering, bioimaging and sensing.
\end{abstract}

\section{Introduction}

Graphene is a novel class of two dimensional mono-layer of carbon atom, which is tightly packed into honey combed lattice. These sp2 hybridized carbon sheets were discovered in 2004 by Giem and Novoselov [1], for which they were awarded the 2010 Nobel Prize in Physics. It is the basic building block of graphitic material of other dimensionalities i.e. it can be wrapped up into $0 \mathrm{D}$ fullerenes, rolled up into 1D carbon nanotubes and stacked one on top of the other into 3D graphite flakes. Ever since the discovery of graphene, it has created waves in the scientific community owing to its unique properties which has an enormous potential to serve a multitude of applications. Due to its specific electrical properties, graphene based sensors are used in nanoelectronics and sensing systems. Graphene and graphene oxide have been explored with many molecular imaging techniques, including magnetic resonance imaging, optical, photoacoustic and radionuclide-based imaging [2-5]. The extraordinary electronic, thermal, optical properties and superior mechanical strength are utilized for biomedical applications as well.

Graphene materials can be broadly classified as graphene family of nanomaterial (GFNs) which includes mono layer graphene, few layer graphene (FLG), ultrathin graphite, graphene oxide (GO), reduced graphene oxide (rGO), graphene quantum dots (GQDs) [6]. Mainly $\mathrm{GO}, \mathrm{rGO}$ and GQDs are used for biomedical purposes like drug delivery, gene delivery, biosensing, bioimaging, tissue engineering and cancer therapy [7-9]. GO is the highly oxidized form of graphene containing carboxyl group $(-\mathrm{COOH})$ in the edges and hydroxyl $(-\mathrm{OH})$ and epoxy (-O-) group in the basal planes, which makes $\mathrm{GO}$ comparatively hydrophilic. On the other hand, rGO is obtained upon reduction of $\mathrm{GO}$ using various reducing agents. It reduces the oxygen content of GO, makes it more hydrophobic, introduces defects in the lattice and also partially restores electrical conductivity. GQDs are nanometer sized particles of graphene exhibiting certain unique properties that are useful for imaging purposes. GQDs are synthesized by top down (decomposition and exfoliation of graphite) or bottom up (synthesis from polycyclic aromatic compounds) method [10].

Functionalization of GFNs is important for biomedical applications. Due to the hydrophobic nature of graphene it is essential to functionalize them to aid in their dispersion. Even though GO is hydrophilic, the charge screening effect in the presence of salts leads to aggregation of these particles in physiological buffer. Moreover, functionalization with appropriate biocompatible coating ameliorates their toxicity and also serves for the controlled release of drug in the biological environment [11]. This can be done using covalent and non covalent strategies. Covalent modification is possible due to the presence of defects in the plane of rGO and GO [12]. The main

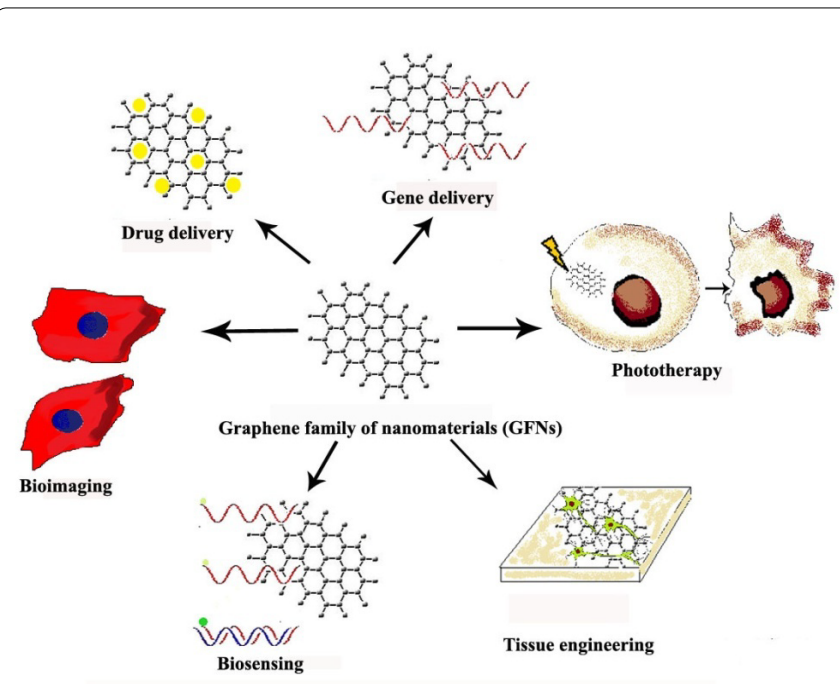

Figure 1: Biomedical application of Graphene family of Nanomaterials (GFNs). 
methods of covalent modification are nucleophilic substitution, electrophilic addition, condensation and addition. Non covalent modification includes Van der Waals forces, electrostatic interation, hydrogen bonding, coordination bonds and $\pi-\pi$ interactions [12].

The fascinating properties of GFNs are channeled for application in drug delivery, gene delivery, photo therapy, tissue engineering, biosensing and bioimaging [13,6,7], as shown in Figure 1. This review will discuss the different graphene based biomedical application and the recent trends in this field.

\section{Drug Delivery}

GFNs are a particularly interesting candidate for drug delivery applications. They have numerous advantageous properties and have been explored in the biomedical field as nano-carriers for the delivery of therapeutic drugs to the biological system. Due to their large surface area, GFNs offers a superior drug loading capacity in comparison to other nano-carriers. Monolayer of graphene presents a unique feature where all the atoms are exposed to the surface and aids in high drug loading capacity [11]. The presence of delocalized $\pi$ electrons allows $\pi-\pi$ stacking with other $\pi$ electron rich drugs such as doxorubicin (DOX) [14]. Non covalent interactions such as electrostatic and hydrophobic interactions also enable loading of poorly soluble drugs on to the graphene platform. GO has a characteristic amphiphilic nature that enables entry through cell membrane. Hydrophilicity arises due to the presence of oxygen containing functional group and numerous unmodified graphenic domains in the plane makes it slightly hydrophobic, thus making it amphiphilic. rGO, which is obtained after reduction of GO is hydrophobic in nature. However, it is characterized by the presence of defect in the lattice and residual oxygen. The liphophilic nature of pristine graphene also facilitates the penetration through biological membrane [5]. Nevertheless pristine graphene needs to be functionalized to make it dispersible before any biomedical application [13]. However despite their numerous unique properties, there are some major limitations. The lateral dimension and shape of graphene limits the entry into the cell and this can also be regarded for their toxicity. Targeted delivery and appropriate functionalization have been employed to overcome these issues.

It is essential to functionalize GFNs before using for drug delivery to aid in dispersion, reduce toxicity and for controlled release of drug. As mentioned above, covalent and non covalent approaches were used in many studies to functionalize GFNs. In one of the initial studies by Sun et al. [15], nano GO was covalently conjugated with PEG and antibody Rituxan (anti-CD20) and then loaded with DOX for targeted killing of cancer cells. More recently Miao and co workers [16] employed EDC chemistry to synthesize PEG grafted GO loaded with anticancer drug DOX and photo sensitizer chlorine e6 (Ce6) for combined chemo and photothermal therapy against cancer cells. In a non-covalent approach, Depan et al. [17] synthesized folate decorated GO for delivering DOX into the cancer cell. DOX, due to the presence of aromatic benzene ring facilitates $\pi-\pi$ stacking with the delocalized $\pi$ electrons present in GO. In another study carried out by Wang et al. [18], cylic RGD modified chitosan and DOX was loaded on to GO using simple non covalent methods and used for drug delivery into hepatocellular carcinomas.

Numerous studies have utilized graphene as a nano carrier for anti cancer drug delivery, targeted drug delivery and controlled drug release. Chemotherapy is one of the major treatments for cancer. However these chemotherapeutic agents adversely affect the health by targeting rapidly dividing cells as well. To overcome that, nanoparticles conjugated with chemotherapeutic agents are under scrutiny. The enhanced permeation and retention (EPR) effect in the tumor sites aid in passive translocation of nanoparticle to the site of the tumor and their retention. Graphene has been loaded with anticancer drugs like DOX [17,19,20], camptothecin [21-23], paclitaxel [24], lucanthone [25] and others [26] successfully. In a study by Zhang et al. [27], nano GO was loaded with two anti cancer drugs- DOX and camptothecin via $\pi-\pi$ interaction. It was then functionalized with folate for uptake via folate receptor pathway in the cancer cell. They found that the particles were successfully taken up by MCF-7 cell lines and showed increased cytotoxicity.

Another main strategy of drug delivery is targeting to the specific site such as tumor, across the blood brain barrier and other specific organs/cells. One of the major focuses of targeted drug delivery is the use of graphene conjugated with ligands such as folic acid [28-30], transferrin [31], monoclonal antibodies [15, 32], RGD peptides [33, 34] and so on. In a recent study, it was shown that GQDs modified with folate and DOX showed enhanced uptake via receptor mediated endocytosis into HeLa cells and showed cytotoxicity [35]. In another novel approach, graphene conjugated with iron oxide nanoparticle was used for magnetic drug delivery to the target in various studies [36-39].

Release of drug in a controlled and sustained manner is one of the important aspects of a drug delivery system, so as to reach a desired therapeutic level at the target site. Zhou et al. [40] formulated a $\mathrm{pH}$ responsive graphene based nanocarrier for the release of DOX. They utilized a charge reversal polyelectrolyte, citraconic anhydridefunctionalized poly (allylamine) (PAH-Cit), which gets cleaved into cationic poly (allylamine) in acidic environment such as that found inside the endosome. DOX was covalently linked to PAH-Cit. PAHCit-DOX was conjugated with polyethyleneimine coated GO. Inside the endosome or lysosome, cleavage of the polyelectrolyte occurred and triggered release of DOX. Others have utilized redox responsive [41] and near infrared (NIR) controlled [42] drug release.

\section{Gene Delivery}

Gene therapy is a promising approach for the treatment of genetic disorders, viral infections as well as for cancer. The major challenge is the development of a safe and efficient gene delivery vector. Ideally the gene delivery vector should protect the nucleic acid from degradation by the nuclease enzyme and have high transfection efficiency. In recent years graphene has been explored as a vehicle for gene delivery into the cells. It has been found that graphene shows a strong attraction towards single stranded DNA (ssDNA) and RNA due to strong $\pi-\pi$ interaction between the nucleic acid bases and the delocalized $\pi$ electrons of graphene. However, it shows less affinity towards the double stranded DNA (dsDNA) as the DNA bases are concealed within the double helix preventing $\pi-\pi$ stacking [43]. In addition to that, it was demonstrated by Lu et al. [44] that graphene could protect oligonucleotides from degradation by cellular nuclease due to steric hindrance effect.

In a study conducted by Kim et al. [45], DNAzyme delivery system based on nano GO was developed for simultaneous sensing and knockdown of hepatitis $C$ viral gene in liver cells. DNAzyme (Dz) is an RNA cleaving oligodeoxyribozyme which mediates catalytic hydrolysis of target mRNA in sequence specific manner. Fluorescence reporter dye FAM was conjugated to Dz and this was complexed with nano GO. The fluorescence of FAM was quenched by a phenomenon called Fluorescence Resonance Energy Transfer (FRET) due to nano GO. The FAM-Dz/nano GO complex was able to enter into liver cells and knockdown viral gene HCV NS3, which is required for viral replication. Moreover, when the duplex is formed between the viral gene and Dz specific for it, the fluorescence of the reporter dye FAM was regained due to desorption from nano GO leading to decreased affinity towards dsDNA. This allows for real-time sensing as well. Zhi et al. [46] used a multifunctional nanocomplex composed of polyethylenimine (PEI)/poly (sodium 4-styrenesulfonates) (PSS)/ GO called PPG for silencing a multidrug resitance gene miR-21. The anticancer drug adriamycin and miR-21 targeted siRNA was loaded into PPG. In vitro experiments on MCF-7 cell line showed that this nano complex enhanced retention of adriamycin and effective silencing of miR-21 gene. In another study, negatively charged GO was bound to cationic PEI by electrostatic attraction forming a stable $\mathrm{GO} / \mathrm{PEI}$ complex rich in positive charge. This enabled the loading of plasmid DNA and successful transfection into HeLa cells [47].

However, there is still a long way to go before graphene vectors could be employed for gene delivery. For this, suitable functionalization 
and improved design of surface chemistry is required, which can pave the way for unique graphene based drug delivery vectors.

\section{Phototherapy}

Phototherapy is one of the emerging forms of treatment for cancer by photo ablation of cells. This is based on two main properties of the photosensitizer upon exposure to light -1 ) generation of reactive oxygen species or ROS (photodynamic therapy, PDT) and 2) generation of heat (photothermal therapy, PTT). Graphene has been investigated for phototherapy owing to its unique properties.

In PDT, photosensitizers absorb light energy and transfer this energy to the surrounding tissue oxygen. This leads to generation of ROS that oxidizes cellular and sub-cellular compartments, finally leading to tumor cell death. However, the success of PDT is limited due to the hydrophobic nature of photosensitizers used. Zhou et al. [48] overcame this by conjugating hyphocrellin, a hydrophobic photosensitizer with GO via non covalent interactions thus enhancing solubility. They found that hypocrellin-GO conjugate was taken up by tumor cells and upon irradiation killed them by generation of singlet oxygen. Miao et al. [16] showed improved efficacy of tumor killing by co-delivery of anticancer drug with photosensitizers immobilized on GO. PEG grafted GO was loaded with DOX and Ce6 photosensitizer by physical adsorption and it was able to kill cancer cell efficiently. Gollavelli and Ling [49] suggested that using photosensitizers having the same NIR absorption range as graphene could facilitate the use of single light phototherapy. They found that immobilizing hydrophobic silicon napthalocyanin bis (trihexylsilyloxide) (SiNc4) photosensitizer on to graphene helped to generate both PDT/ PTT effects simultaneously using a single wavelength of light and effectively killing the tumor cell.

GO and rGO has optical absorption at the NIR region. In PTT, upon exposure to laser, the material can generate heat and kill cells by photo ablation. In one of the initial studies by Yang et al. [50], they utilized this property of graphene for killing tumor cells in vivo. Various tumor models were created in Balb/c mice and they were injected with PEGylated nanographene sheets. They showed passive tumor targeting and retention and showed no significant signs of toxicity in the animals. Upon laser irradiation at a power density of $2 \mathrm{~W} / \mathrm{cm}^{2} 24 \mathrm{~h}$ after injection, it was seen that the temperature rose to $\sim 50^{\circ} \mathrm{C}$ at the tumor site. It was seen that all irradiated tumor in mice injected with nano graphene sheets disappeared completely within 1 week. Zhang et al. [51] used a combination of chemo-phototherapy for killing cancer cells using graphene. DOX was loaded onto PEGylated nano GO and used to kill tumor in mice. They found that laser irradiation in addition to the chemotherapeutic effect of DOX, aided in $100 \%$ tumor elimination in all the animals. In another interesting study, dissociation of amyloid- $\beta$ fibrils, a hallmark of Alzheimer's disease, was facilitated upon exposure to nano GO and subsequent irradiation [52]. This study provides insight into designing graphene based system for phothermal therapy of Alzheimer's disease. Nurunnabi et al. [53] showed that carboxylated photoluminescent graphene nanodots (cGdots) can be used for phototherapy through PTT and PDT and also facilitate imaging of tumor. They found that upon laser irradiation, electrons of cGdots start to vibrate and form an electron cloud, thereby generating sufficient heat to kill cancer cell by PTT. In addition to that, cGdots having high level of unsaturation, absorb light and transfer the energy to surrounding oxygen molecules and unsaturated bonds. This results in generation of ROS and subsequently kills cancer cells (PDT).

\section{Tissue Engineering}

Tissue engineering has emerged as an alternative approach for the treatment of lost or malfunctioning organs. It is a multifunctional field that applies the knowledge of engineering and biosciences to develop a biological substitute that aids in regeneration of the damaged tissue. In tissue engineering, a scaffold acts as an artificial extra-cellular matrix (ECM) for the cells to adhere, migrate, proliferate and differentiate on. Ideally a tissue engineered scaffold should promote cell growth and proliferation, should be biocompatible with the system, should be biodegradable, should be porous enough for cells to adhere and form ECM and should be mechanically strong [54]. Recently the prospect of graphene in regeneration therapy, wound healing and stem cell therapy has been explored. Graphene has superior mechanical strength which can be incorporated into various scaffolds to increase the strength. A single layer of defect free graphene is 200 times stronger than steel. In a single layer defect free graphene, the Young's modulus is $1000 \mathrm{GPa}$ whereas fracture strength and Poisson's ratio are $130 \mathrm{GPa}$ and $0.149 \mathrm{GPa}$ respectively $[55,56]$. Due to its superior mechanical strength, graphene has been used to reinforce polymeric scaffolds. The Young's modulus, tensile strength and elastic modulus of graphene reinforced chitosan was significantly high when compared to chitosan alone $[57,58]$. Sun and $\mathrm{Wu}$ [59] developed a thermal and $\mathrm{pH}$ responsive hydrogel using GO covalently linked to poly (N-isopropylacrylamide) (PNIPAM). The cross linking reaction was found to increase the mechanical strength of the GO/PNIPAM hydrogel.

The electrical conductivity of graphene is beneficial for cells that require electrical stimulation for growth and differentiation like neurons. Graphene is a zero band gap semi conductor. Electrons in defect free graphene behave as massless dirac fermions and hence can travel sub-micrometer distances by a phenomenon called ballistic transport [1]. This makes graphene a superior electrical conductor. The electrical conductivity of single defect free graphene is $10^{4} \mathrm{~S} /$ $\mathrm{cm}$ and that of graphene oxide is $10^{-1} \mathrm{~S} / \mathrm{cm}$ at room temperature [60]. Human neural stem cells (hNSC) are being used for neural regeneration and brain repair and it is essential to direct their differentiation towards neuronal cell rather than glial lineage. Improved differentiation and proliferation of hNSCs was found when cultured in a graphene substrate [61,62]. Park et al. [61] found that neuronal marker Beta III tubulin (TUJ1) showed significantly increased expression that glial cell marker (Glial fibrillary acidic protein or GFAP), when grown over graphene substrate. It was also found that there was an increase in laminin related cell adhesion molecule receptor, which enhanced the adhesion of cells to substrate [61]. Graphene being an excellent electrode was found to deliver electrical currents which can be used for neural stimulation and differentiation [62]. Similarly in mouse hippocampal neurons cultured over a graphene substrate, neurite outgrowth was promoted and increased expression of growth associated protein -43 (GAP-43), which is involved in the neurite formation and regeneration, was noticed $[63,64]$. Additionally when rat cortical neurons were plated on to graphene and poly D lysine coated tissue culture polystyrene dishes, no cytotoxicity was noticed [65]. Recent advancements in this field utilized 3D foams coated with graphene by chemical vapour deposition technique and favourable growth and differentiation of NSCs were observed [66]. Taken together, graphene provides improved cell attachment, biocompatibility and neural stimulation for growth and differentiation of neurons. These properties can be harnessed for making novel neural prosthetic devices.

The intrinsic unique properties have also been utilized in stem cell therapy. Nayak et al. [67] investigated osteogenic differentiation potential of human mesenchymal stem cells (hMSCs) grown on graphene coated over different substrates. They found that the cell growth and viability remained unaffected showing biocompatibility. Moreover, hMSCs grown over graphene were able to differentiate into osteogenic lineage in the absence of BMP-2 growth factor, which was evidenced by the expression of osteocalcin (marker for osteoblasts). Numerous other reports also show evidence of osteogenic differentiation potential of stem cells grown over graphene [68-70]. Graphene has also shown ability to induce differentiation in induced pluripotent stem cells (iPSCs) towards endodermal lineage [71], stimulated differentiation of hMSCs to adipocytes [72], induce myoblasts to form myotube [73] and differentiate hNSCs to neurons [61,66] and oligodendrocytes [74]. Lee et al. [75] proved that the reason for accelerated stem cell adhesion, proliferation and differentiation can be attributed to the mechanical and chemical properties of graphene. 
The osteogenic differentiation potential is traced to the ability of graphene and GO to preconcentrate osteogenic inducers like dexamethasone and $\beta$-glycerolphosphate. Also it was found that GO promoted adipogenesis of MSCs because of its high affinity towards insulin, which is a main mediator of fatty acid synthesis. On the other hand, graphene induced denaturation of insulin due to $\pi-\pi$ interaction hindering adipose differentiation. Also it was suggested that stem cells subjected to physical stress as a result of the nano-topography can enhance differentiation of stem cell to a specific lineage. The presence of ripples and folds on the surface of graphene can therefore be a cue to stem cell differentiation [73-75]. Furthermore, it was suggested that graphite oxide having a size greater than $20 \mathrm{~nm}$ at a concentration of $0.005 \mathrm{mg} / \mathrm{ml}$ was found to be biocompatible with mouse embryonic stem cells (ESCs) and can be used for tissue engineering purposes [76].

In addition to that, graphene and $\mathrm{GO}$ exhibits antimicrobial activity, that is essential for wound healing applications. It has been found that the antibacterial property is the result of synergistic effect of membrane disruption and the oxidative stress caused by the graphene particles. The sharp edges of graphene can perturb the membrane and cause leakage of cellular contents, eventually leading to cell death [77]. Liu et al. [77] also suggested that graphite, graphite oxide, GO and rGO can be internalized by bacterial cells and cause oxidation of GSH leading to oxidative stress in bacteria. Lu et al. [78] used graphene doped chitosan nanofibers for wound healing purposes. Presence of microbes in the wound can lead to delayed wound healing. Hence graphene was used as an antimicrobial agent They proposed that electrons from graphene can easily enter and damage the DNA of prokaryotic cells whereas the eukaryotic DNA is protected by the nuclear membrane, thus preventing DNA damage. It was also found by Sun et al. [79] that GQDs have peroxidase like activity that converts hydrogen peroxide to hydroxyl radical. This too aids in enhancing the antibacterial property and can be used for wound disinfection. However, it was found by Chen et al. [80] that graphene oxide shows a broad spectrum antibacterial and antifungal activity by membrane perturbation and subsequent leakage of electrolytes in the cell and cell death. The mechanism of graphene interaction and toxicity to prokaryotic and eukaryotic pathogens need to be understood further. Nonetheless, GFNs appears to be a promising candidate for tissue engineering, stem cell therapy and wound healing purposes.

\section{Biosensor}

A biosensor is used for the accurate, sensitive and selective detection of analyte or biomolecule. This helps in the diagnosis of genetic disorders, diseases, contaminations, microbial detection and so on. GFNs are versatile and can be modified aptly for the desired biosensing application. Due to its planar conformation graphene provides a large platform for the interaction of probe and analyte with minimum noise. Graphene based biosensors make use of two strategies-1) probe molecule on graphene interacts with the analyte (FRET based biosensing) and 2) a label free technique based on the change in electrical property of graphene when it interacts with the analyte (electrochemical biosensing) [81]

Electrochemical sensing using graphene is made possible due to a phenomenon called Heterogenous electron transfer (HET) that occurs at the edge and defect sight in the basal plane of graphene. When graphene interacts with an analyte, electrons are transferred from the graphene to the analyte for oxidation/reduction of the same, which can be detected [82]. Song et al. [83] synthesized GO based $\mathrm{CuO}$ nanocomposite electrode for the sensitive detection of glucose by non enzymatic method. The $\mathrm{Cu}$ (II)/Cu (III) redox couple is essential for the electrochemical detection of glucose, where electrons are transferred from glucose to $\mathrm{Cu}$. Graphene serves as a conducting substrate enhancing sensitivity and accuracy. In a study by Kang et al. [84], glucose oxidase was immobilized on graphene-chitosan nanoplatform and used for the electrochemical sensing of glucose molecules. Glucose oxidase maintained its native structure and higher loading capacity of glucose oxidase was possible on graphene.
The higher sensitivity and accuracy of this biosensing platform was attributed to large surface to volume ratio and good conductivity of graphene. A more extensive discussion on the graphene based electrochemical biosensors is reviewed by Fan and Wang [85]

Graphene based fluorescent biosensing has also been extensively researched due to the fluorescent quenching ability of graphene, called Fluorescence Resonance Energy Transfer (FRET). Graphene contains delocalized $\pi$ electrons that allow for accepting energy from nearby molecules. Hence it can be an excellent quencher of electronically excited molecules [86]. In one of the earlier studies, thrombin detection was facilitated by an aptamer loaded on graphene by $\pi-\pi$ stacking [87]. The aptamer, which is specific for thrombin was conjugated with FAM, a fluorescent molecule. Loading of FAMaptamer onto graphene ensured close proximity of the fluorescent dye to graphene which enables FRET from dye to graphene. This allows efficient fluorescence quenching. The presence of thrombin induces weak binding and conformation change in the aptamer and subsequently the dye moves far away from graphene enabling fluorescence recovery, thus aiding in thrombin detection. Similarly, in 2013, Hu et al. [88] developed graphene based aptasensor for detection of IgE. They were able to detect IgE at a range of 60-225 pM and found that the detection limit was $22 \mathrm{pM}$. This was found to be extremely sensitive when compared to the current detection methods which has detection limit up to $2 \mathrm{nM}$.

Although the potential of graphene based biosensors are numerous, it still requires extensive study and fabrication before use in in vivo and in vitro detection.

\section{Bioimaging}

The excellent optical properties of GFNs make it an appealing candidate for bioimaging purposes. It was shown by Sun et al. [15] that GO exhibited photoluminescence (PL) at the NIR region, which is useful for cellular imaging purposes due to the minimal cellular auto-fluorescence in this region. Kim et al. [43] developed GO -polyethylenimine nano-construct for the simultaneously gene delivery and bioimaging. The PL property of GO is also due to the presence of isolated poly aromatic structures. However, the presence of carboxylic and epoxide functional group on GO induce nonradiative recombination of electron-hole pair. This can cause low emission in GO. Kim et al. [43] overcame this by conjugating GO with polyethelenimine which restores its aromatic domains.

GQDs are nanometer sized fragments of graphene which show unique electrical and optical properties. GQDs shows quantum confinement effect, which results in a non zero band gap and luminescence on excitation [10]. Zhu et al. [89] synthesized GQDs by solvothermal methods and demonstrated their PL and biocompatibility. They suggested that surface defect in addition to quantum confinement contributed to the photoluminescence of GQDs. In a recent study, Nahain et al. [90] used GQDs conjugated with hyaluronic acid for specific targeting and imaging of CD44 over expressed tumor in vivo. Hyaluronic acid functionalized GQDs labeled human serum albumin were used to image pancreatic cancer cells in vitro [91]. However, some of the drawbacks of using GQDs for practical biological application are - 1) the use of one-photon UV excitation which causes unavoidable damage to the biological sample, 2) PL of GQD falls near the visible range, which is near to the background of biological sample, making it impossible to distinguish signals emitted by them and 3) PL of most GQDs depends upon $\mathrm{pH}$ and ionic strength making them difficult to use in quantitative assays [92]. So to address these shortcomings, Chen et al. [92] developed pyrrole-ring modified GQDs which have an excitation wavelength of $490 \mathrm{~nm}$ and an emission wavelength of $550 \mathrm{~nm}$. It was found to be stable at $\mathrm{pH} 4-10$ and ionic strength $1.2 \mathrm{molL}^{-1} \mathrm{KCl}$ and the PL emission was found to be excitation independent.

Hence these intrinsic properties of GFNs open new avenues of research in the imaging field. However the research is still in its infancy and has to go a long way before it can be marketed. 


\section{Conclusion}

GFNs have excited the scientific community over the past decade since their discovery. The unique electronic properties, superior mechanical strength, thermal properties and distinctive optical properties have been harnessed in the biomedical field as well as in nanoelectronics. However, there are still miles to go before any graphene based product can be commercialized. The reports on toxicity of graphene family of nanomaterial are highly inconsistent. This might be due to the difference in method of synthesis, raw materials used, cell system used and so on. So a thorough biological evaluation and standards are required for elucidating their interaction with the biological system. Also the pharmacokinetics and biodegradation of GFNs, on entering the body, needs to be well characterized before commercialization. This paves way for innovative new research to understand their interaction with the biological system and also to tailor specific and biocompatible products for biomedical approaches.

\section{References}

1. Novoselov KS, Geim AK, Morozov SV, Jiang D, Zhang Y, et al. (2004) Electric field effect in atomically thin carbon films. Science 306: 666-669.

2. Ruoff R (2008) Graphene: calling all chemists. Nat Nanotechnol 3: 10-11.

3. Hong $\mathrm{H}$, Yang K, Zhang Y, Engle JW, Feng L, et al. (2012) In vivo targeting and imaging of tumor vasculature with radiolabeled, antibody-conjugated nanographene. ACS Nano 6: 2361-2370.

4. Hong $\mathrm{H}$, Zhang $\mathrm{Y}$, Engle JW, Nayak TR, Theuer CP, et al. (2012) In vivo targeting and positron emission tomography imaging of tumor vasculature with (66)Ga-labeled nano-graphene. Biomaterials 33: 4147-4156.

5. Novoselov KS, Fal'ko VI, Colombo L, Gellert PR, Schwab MG, et al. (2012) A roadmap for graphene. Nature 490: 192-200.

6. Sanchez VC, Jachak A, Hurt RH, Kane AB (2012) Biological interactions of graphene-family nanomaterials: an interdisciplinary review. Chem Res Toxicol 25: 15-34.

7. Shen H, Zhang L, Liu M, Zhang Z (2012) Biomedical applications of graphene Theranostics 2: 283-294.

8. Zhang Y, Nayak TR, Hong H, Cai W (2012) Graphene: a versatile nanoplatform for biomedical applications. Nanoscale 4: 3833-3842.

9. Wang Y, Li Z, Wang J, Li J, Lin Y (2011) Graphene and graphene oxide: biofunctionalization and applications in biotechnology. Trends Biotechnol 29: 205-212.

10. Bacon M, Bradley SJ, Nann T (2013) Graphene Quantum Dots. Part Part Syst Charact 31: 415-428.

11. Liu J, Cui L, Losic D (2013) Graphene and graphene oxide as new nanocarriers for drug delivery applications. Acta Biomater 9: 9243-9257.

12. Georgakilas V, Otyepka M, Bourlinos AB, Chandra V, Kim N, et al. (2012) Functionalization of graphene: covalent and non-covalent approaches, derivatives and applications. Chem Rev 112: 6156-6214.

13. Stankovich S, Dikin DA, Dommett GH, Kohlhaas KM, Zimney EJ, et al. (2006) Graphene-based composite materials. Nature 442: 282-286.

14. Yang X, Zhang X, Liu Z, Ma Y, Huang Y, et al. (2008) High-Efficiency Loading and Controlled Release of Doxorubicin Hydrochloride on Graphene Oxide. J Phys Chem C 112: 17554-17558.

15. Sun X, Liu Z, Welsher K, Robinson JT, Goodwin A, et al. (2008) NanoGraphene Oxide for Cellular Imaging and Drug Delivery. Nano Res 1: 203 212

16. Miao W, Shim G, Lee S, Lee S, Choe YS, et al. (2013) Safety and tumor tissue accumulation of pegylated graphene oxide nanosheets for co-delivery of anticancer drug and photosensitizer. Biomaterials 34: 3402-3410.

17. Depan D, Shah J, Misra RDK (2011) Controlled release of drug from folatedecorated and graphene mediated drug delivery system: Synthesis, loading efficiency, and drug release response. Materials Science and Engineering C. $31: 1305-1312$

18. Wang C, Chen B, Zou M, Cheng G (2014) Cyclic RGD-modified chitosan/ graphene oxide polymers for drug delivery and cellular imaging. Colloids Surf B Biointerfaces 122: 332-340.

19. Yang X, Wang $Y$, Huang $X$, Ma $Y$, Huang $Y$, et al. (2010) Multi-functionalized graphene oxide based anticancer drug-carrier with dual-targeting function and pH-sensitivity. J Mater Chem 21: 3448-3454

20. Zhao X, Liu L, Li X, Zeng J, Jia X, et al. (2014) Biocompatible graphene oxide nanoparticle-based drug delivery platform for tumor microenvironmentresponsive triggered release of Doxorubicin. Langmuir. 30: 10419-10429.
21. Sahoo NG, Bao H, Pan Y, Pal M, Kakran M, et al. (2011) Functionalized carbon nanomaterials as nanocarriers for loading and delivery of a poorly water-soluble anticancer drug: a comparative study. Chem Commun (Camb) 47: 5235-5237.

22. Bao H, Pan Y, Ping Y, Sahoo NG, Wu T, et al. (2011) Chitosan-functionalized graphene oxide as a nanocarrier for drug and gene delivery. Small 7: 15691578.

23. Saikia N, Deka RC (2013) Ab initio study on the noncovalent adsorption of camptothecin anticancer drug onto graphene, defect modified graphene and graphene oxide. J Comput Aided Mol Des 27: 807-821.

24. Xu Z, Wang S, Li Y, Wang M, Shi P, et al. (2014) Covalent Functionalization of Graphene Oxide with Biocompatible Poly(ethylene glycol) for Delivery of Paclitaxel. ACS Appl Mater Interfaces 6: 17268-17276.

25. Chowdhury SM, Surhland C, Sanchez Z, Chaudhary P, Suresh Kumar MA et al. (2014) Graphene nanoribbons as a drug delivery agent for lucanthone mediated therapy of glioblastoma multiforme. Nanomedicine 1549-9634: 00424-00429.

26. Chen J, Liu H, Zhao C, Qin G, Xi G, et al. (2014) One-step reduction and PEGylation of graphene oxide for photothermally controlled drug delivery. Biomaterials 35: 4986-4995

27. Zhang L, Xia J, Zhao Q, Liu L, Zhang Z (2010) Functional graphene oxide as a nanocarrier for controlled loading and targeted delivery of mixed anticancer drugs. Small 6: 537-544.

28. Maity AR, Chakraborty A, Mondal A, Jana NR (2014) Carbohydrate coated folate functionalized colloidal graphene as a nanocarrier for both hydrophobic and hydrophilic drugs. Nanoscale 6: 2752-2758.

29. Qin XC, Guo ZY, Liu ZM, Zhang W, Wan MM, et al. (2013) Folic acidconjugated graphene oxide for cancer targeted chemo-photothermal therapy. J Photochem Photobiol B 120: 156-162.

30. Wang Z, Zhou C, Xia J, Via B, Xia Y, et al. (2013) Fabrication and characterization of a triple functionalization of graphene oxide with $\mathrm{Fe} 3 \mathrm{O} 4$, folic acid and doxorubicin as dual-targeted drug nanocarrier. Colloids Surf B Biointerfaces 106: 60-65.

31. Liu G, Shen H, Mao J, Zhang L, Jiang Z, et al. (2013) Transferrin modified graphene oxide for glioma-targeted drug delivery: in vitro and in vivo evaluations. ACS Appl Mater Interfaces 5: 6909-6914.

32. Yang HW, Lu YJ, Lin KJ, Hsu SC, Huang CY, et al. (2013) EGRF conjugated PEGylated nanographene oxide for targeted chemotherapy and photothermal therapy. Biomaterials 34: 7204-7214.

33. Wang C, Chen B, Zou M, Cheng G (2014) Cyclic RGD-modified chitosan/ graphene oxide polymers for drug delivery and cellular imaging. Colloids Surf B Biointerfaces 122: 332-340.

34. Dong H, Li Y, Yu J, Song Y, Cai X, et al. (2013) A versatile multicomponent assembly via $\hat{~}^{2}$-cyclodextrin host-guest chemistry on graphene for biomedical applications. Small 9: 446-456.

35. Wang X, Sun X, Lao J, He H, Cheng T, et al. (2014) Multifunctional graphene quantum dots for simultaneous targeted cellular imaging and drug delivery. Colloids Surf B Biointerfaces 122: 638-644

36. Yang X, Zhang X, Ma Y, Huang Y, Wang Y, et al. (2009) Superparamagnetic graphene oxide-Fe3O4nanoparticles hybrid for controlled targeted drug carriers. J Mater Chem 19: 2710-2714.

37. Yang X, Wang Y, Huang X, Ma Y, Huang Y, et al. (2010) Multi-functionalized graphene oxide based anticancer drug-carrier with dual-targeting function and $\mathrm{pH}$-sensitivity. J Mater Chem 21: 3448-3454.

38. Ma X, Tao H, Yang K, Feng L, Cheng L, et al. (2012) A functionalized graphene oxide-iron oxide nanocomposite for magnetically targeted drug delivery, photothermal therapy, and magnetic resonance imaging. Nano Res 5: 199-212.

39. Fan X, Jiao G, Zhao W, Jin P, Li X (2013) Magnetic Fe3O4-graphene composites as targeted drug nanocarriers for $\mathrm{pH}$-activated release. Nanoscale 5: 1143-1152

40. Zhou T, Zhou X, Xing D (2014) Controlled release of doxorubicin from graphene oxide based charge-reversal nanocarrier. Biomaterials 35: 41854194.

41. Wen H, Dong C, Dong $H$, Shen A, Xia W, et al. (2012) Engineered redoxresponsive PEG detachment mechanism in PEGylated nano-graphene oxide for intracellular drug delivery. Small 8: 760-769.

42. Kurapati R, Raichur AM (2013) Near-infrared light-responsive graphene oxide composite multilayer capsules: a novel route for remote controlled drug delivery. Chem Commun (Camb) 49: 734-736.

43. Kim H, Namgung R, Singha K, Oh IK, Kim WJ (2011) Graphene oxidepolyethylenimine nanoconstruct as a gene delivery vector and bioimaging tool. Bioconjug Chem 22: 2558-2567.

44. Lu CH, Zhu CL, Li J, Liu JJ, Chen X, et al. (2010) Using graphene to protect 
DNA from cleavage during cellular delivery. Chem Commun (Camb) 46: 3116-3118.

45. Kim S, Ryoo SR, Na HK, Kim YK, Choi BS, et al. (2013) Deoxyribozymeloaded nano-graphene oxide for simultaneous sensing and silencing of the hepatitis $C$ virus gene in liver cells. Chem Commun (Camb) 49: 8241-8243.

46. Zhi F, Dong H, Jia X, Guo W, Lu H, et al. (2013) Functionalized graphene oxide mediated adriamycin delivery and miR-21 gene silencing to overcome tumor multidrug resistance in vitro. PLoS One 8: e60034.

47. Feng L, Zhang S, Liu Z (2011) Graphene based gene transfection. Nanoscale 3: $1252-1257$.

48. Zhou L, Wang W, Tang J, Zhou JH, Jiang HJ, et al. (2011) Graphene oxide noncovalent photosensitizer and its anticancer activity in vitro. Chemistry 17 12084-12091.

49. Gollavelli G, Ling YC2 (2014) Magnetic and fluorescent graphene for dua modal imaging and single light induced photothermal and photodynamic therapy of cancer cells. Biomaterials 35: 4499-4507.

50. Yang K, Zhang S, Zhang G, Sun X, Lee ST, et al. (2010) Graphene in mice: ultrahigh in vivo tumor uptake and efficient photothermal therapy. Nano Lett 10: $3318-3323$

51. Zhang W, Guo Z, Huang D, Liu Z, Guo X, et al. (2011) Synergistic effect of chemo-photothermal therapy using PEGylated graphene oxide. Biomaterials 32: $8555-8561$.

52. Li M, Yang X, Ren J, Qu K, Qu X (2012) Using graphene oxide high nearinfrared absorbance for photothermal treatment of Alzheimer's disease. Adv Mater 24: 1722-1728.

53. Nurunnabi M, Khatun Z, Reeck GR, Lee DY, Lee YK (2014) Photoluminescent graphene nanoparticles for cancer phototherapy and imaging. ACS App Mater Interfaces 6: 12413-12421.

54. Chen G, Ushida T, Tateishi T (2000) Scaffold Design for Tissue Engineering Macromol Biosci 2:67-77.

55. Li JL, Kudin KN, McAllister MJ, Prud'homme RK, Aksay IA, et al. (2006) Oxygen-driven unzipping of graphitic materials. Phys Rev Lett 96: 176101.

56. Goenka S, Sant V, Sant S (2014) Graphene-based nanomaterials for drug delivery and tissue engineering. J Control Release 173: 75-88.

57. Fan H, Wang L, Zhao K, Li N, Shi Z, et al. (2010) Fabrication, mechanical properties, and biocompatibility of graphene-reinforced chitosan composites. Biomacromolecules 11: 2345-2351.

58. Yang X, Tu Y, Li L, Shang S, Tao XM (2010) Well-dispersed chitosan graphene oxide nanocomposites. ACS Appl Mater Interfaces 2: 1707-1713.

59. Sun S, Wu P (2011) A one-step strategy for thermal- and $\mathrm{pH}$-responsive graphene oxide interpenetrating polymer hydrogel networks. J Mater Chem 21: 4095-4097.

60. Gao W, Alemany LB, Ci L, Ajayan PM (2009) New insights into the structure and reduction of graphite oxide. Nat Chem 1: 403-408.

61. Park SY, Park J, Sim SH, Sung MG, Kim KS, et al. (2011) Enhanced differentiation of human neural stem cells into neurons on graphene. Adv Mater 23: H263-267.

62. Tang M, Song Q, Li N, Jiang Z, Huang R, et al. (2013) Enhancement of electrical signaling in neural networks on graphene films. Biomaterials 34 : 6402-6411.

63. Li N, Zhang X, Song Q, Su R, Zhang Q, et al. (2011) The promotion of neurite sprouting and outgrowth of mouse hippocampal cells in culture by graphene substrates. Biomaterials 32: 9374-9382.

64. Tu Q, Pang L, Wang L, Zhang Y, Zhang R, et al. (2013) Biomimetic cholinelike graphene oxide composites for neurite sprouting and outgrowth. ACS Appl Mater Interfaces 5: 13188-13197.

65. Sahni D, Jea A, Mata JA, Marcano DC, Sivaganesan A, et al. (2013) Biocompatibility of pristine graphene for neuronal interface. J Neurosurg: Pediatr 11: 575-583.

66. Li N, Zhang Q, Gao S, Song Q, Huang R, et al. (2013) Three-dimensional graphene foam as a biocompatible and conductive scaffold for neural stem cells. Sci Rep 3: 1604.

67. Nayak TR, Andersen H, Makam VS, Khaw C, Bae S, et al. (2011) Graphene for controlled and accelerated osteogenic differentiation of human mesenchymal stem cells. ACS Nano 5: 4670-4678.

68. Crowder SW, Prasai D, Rath R, Balikov DA, Bae H, et al. (2013) Threedimensional graphene foams promote osteogenic differentiation of human mesenchymal stem cells. Nanoscale 5: 4171-4176.

69. Tatavarty R, Ding H, Lu G, Taylor RJ, Bi X (2014) Synergistic acceleration in the osteogenesis of human mesenchymal stem cells by graphene oxidecalcium phosphate nanocomposites. Chem Commun (Camb) 50: 8484-8487.

70. Elkhenany H, Amelse L, Lafont A, Bourdo S, Caldwell M, et al. (2014)
Graphene supports in vitro proliferation and osteogenic differentiation of goat adult mesenchymal stem cells: potential for bone tissue engineering. J Appl Toxicol

71. Chen GY, Pang DW, Hwang SM, Tuan HY, Hu YC (2012) A graphenebased platform for induced pluripotent stem cells culture and differentiation. Biomaterials 33: 418-427.

72. Kim J, Choi KS, Kim Y, Lim KT, Seonwoo H, et al. (2013) Bioactive effects of graphene oxide cell culture substratum on structure and function of human adipose-derived stem cells. J Biomed Mater Res A 101: 3520-3530.

73. Ku SH, Park CB (2013) Myoblast differentiation on graphene oxide. Commons below Biomaterials 34: 2017-2023.

74. Shah S, Yin PT, Uehara TM, Chueng ST, Yang L, et al. (2014) Guiding stem cell differentiation into oligodendrocytes using graphene-nanofiber hybrid scaffolds. Adv Mater 26: 3673-3680.

75. Lee WC, Lim CH, Shi H, Tang LA, Wang Y, et al. (2011) Origin of enhanced stem cell growth and differentiation on graphene and graphene oxide. ACS Nano 5: 7334-7341.

76. Wang IN, Robinson JT, Do G, Hong G, Gould DR, et al. (2014) Graphite oxide nanoparticles with diameter greater than $20 \mathrm{~nm}$ are biocompatible with mouse embryonic stem cells and can be used in a tissue engineering system. Small 10: 1479-1484.

77. Liu S, Zeng TH, Hofmann M, Burcombe E, Wei J, et al. (2011) Antibacterial activity of graphite, graphite oxide, graphene oxide, and reduced graphene oxide: membrane and oxidative stress. ACS Nano 5: 6971-6980.

78. Lu B, Li T, Zhao H, Li X, Gao C, et al. (2012) Graphene-based composite materials beneficial to wound healing. Nanoscale 4: 2978-2982.

79. Sun H, Gao N, Dong K, Ren J, Qu X (2014) Graphene quantum dots-bandAIDS used for wound disinfection. ACS Nano 8: 6202-6210.

80. Chen J, Peng H, Wang X, Shao F, Yuan Z, et al. (2014) Graphene oxide exhibits broad-spectrum antimicrobial activity against bacterial phytopathogens and fungal conidia by intertwining and membrane perturbation. Nanoscale 6 : 1879-1889.

81. Bitounis D, Ali-Boucetta H, Hong BH, Min DH, Kostarelos K (2013) Prospects and challenges of graphene in biomedical applications. Adv Mater 25: 22582268 .

82. Pumera M (2011) Graphene in biosensing. Materials today 14: 308-315

83. Song J, Xu L, Zhou C, Xing R, Dai Q, et al. (2013) Synthesis of Graphene Oxide Based CuO Nanoparticles Composite Electrode for Highly Enhanced Nonenzymatic Glucose Detection. ACS Appl Mater Interfaces 5: 1292812934

84. Kang X, Wang J, Wu H, Aksay IA, Liu J, et al. (2009) Glucose oxidasegraphene-chitosan modified electrode for direct electrochemistry and glucose sensing. Biosens Bioelectron 25: 901-905.

85. Fang Y, Wang E (2013) Electrochemical biosensors on platforms of graphene. Chem Commun (Camb) 49: 9526-9539.

86. Feng L, Liu Z (2011) Graphene in biomedicine: opportunities and challenges. Nanomedicine (Lond) 6: 317-324.

87. Chang H, Tang L, Wang Y, Jiang J, Li J (2010) Graphene fluorescence resonance energy transfer aptasensor for the thrombin detection. Anal Chem 82: 2341-2346.

88. Hu K, Yang H, Zhou J, Zhao S, Tian J (2013) Aptasensor for amplified IgE sensing based on fluorescence quenching by graphene oxide. Luminescence 28: $662-666$.

89. Zhu S, Zhang J, Qiao C, Tang S, Li Y, et al. (2011) Strongly greenphotoluminescent graphene quantum dots for bioimaging applications. Chem Commun (Camb) 47: 6858-6860.

90. Abdullah-Al-Nahain, Lee JE, In I, Lee H, Lee KD, et al. (2013) Target delivery and cell imaging using hyaluronic acid-functionalized graphene quantum dots. Mol Pharm 10: 3736-3744

91. Nigam P, Waghmode S, Louis M, Wangnoo S, Chavan P, et al. (2014) Graphene quantum dots conjugated albumin nanoparticles for targeted drug delivery and imaging of pancreatic cancer. J Mater Chem B 2: 3190-3195.

92. Chen S, Hai X, Xia C, Chen XW, Wang JH (2013) Preparation of excitationindependent photoluminescent graphene quantum dots with visible-light excitation/emission for cell imaging. Chemistry 19: 15918-15923. 